\title{
Detection of Motion-defined form using Night Vision Goggles
}

\author{
Todd Macuda*a ${ }^{\mathrm{a}}$, Greg Craig ${ }^{\mathrm{a}}$ Robert S. Allison ${ }^{\mathrm{b}}$, Pearl Guterman ${ }^{\mathrm{b}}$, Paul Thomas ${ }^{\mathrm{c}}$, Sion Jennings ${ }^{\mathrm{a}}$ \\ ${ }^{a}$ National Research Council, Flight Research Laboratory, Ottawa, Canada K1A 0R6; \\ ${ }^{b}$ York University, Department of Computer Science, Toronto, Canada, M3J 1P3 \\ ${ }^{\mathrm{c}}$ Topaz Technology Inc., Toronto, Canada
}

\begin{abstract}
Perception of motion-defined form is important in operational tasks such as search and rescue and camouflage breaking. Previously, we used synthetic Aviator Night Vision Imaging System (ANVIS-9) imagery to demonstrate that the capacity to detect motion-defined form was degraded at low levels of illumination (see Macuda et al., 2004; Thomas et al., 2004). To validate our simulated NVG results, the current study evaluated observer's ability to detect motion-defined form through a real ANVIS-9 system. The image sequences consisted of a target (square) that moved at a different speed than the background, or only depicted the moving background. For each trial, subjects were shown a pair of image sequences and required to indicate which sequence contained the target stimulus. Mean illumination and hence image noise level was varied by means of Neutral Density (ND) filters placed in front of the NVG objectives. At each noise level, we tested subjects at a series of target speeds. With both real and simulated NVG imagery, subjects had increased difficulty detecting the target with increased noise levels, at both slower and higher target speeds. These degradations in performance should be considered in operational planning. Further research is necessary to expand our understanding of the impact of NVG-produced noise on visual mechanisms.
\end{abstract}

Keywords: night vision goggles, visual perception, apparent motion, motion-defined form, psychophysics

\section{INTRODUCTION}

\subsection{Background: Night Vision Goggles influence visual perception.}

Night Vision Goggles (NVGs) allow pilots to see and navigate under minimal levels of illumination by amplifying the available light. However, they have been shown to influence visual perception and may be counted as a causal factor in military helicopter incidents and accidents in a number of countries. ${ }^{1,2}$ For example, the electro-optical characteristics of NVGs have been shown to influence visual perception and image quality. ${ }^{3}$ While the NVGs amplify available light, under very low light conditions, they also create scintillating noise (i.e. a 'grainy' appearance similar to a detuned television) within the visual display that may influence depth, motion, resolution, form, size and distance perception. This degradation in image quality may produce a decrease in aviator performance and an increase in the workload.

\subsection{NVG image noise and visual perception.}

A reduction in the input light level increases the NVG noise, which can lead to impaired visual processing. ${ }^{4,5,6,7,8,9,10,}$

${ }^{11}$ In brief, previous research has shown that visual processes such as texture perception are unaffected while motion and visual acuity are impaired as noise levels increase. Although several groups have been working on perceptually characterizing NVG image noise, the research thrust of this laboratory has been to physically characterize noise and link it to visual processing (e.g. motion perception). ${ }^{9,}{ }^{10,11}$ In keeping with this approach, our previous efforts with synthetic imagery showed that the capacity to detect motion-defined form is impaired under low levels of illumination.

* Todd.Macuda@nrc-cnrc.gc.ca; phone 1613 998-3014; fax 1613 952-1704 
To validate these findings we compared the capacity to detect motion-defined form under simulated and real NVG conditions.

\section{METHODS}

\subsection{Participants}

Two observers (1 male, 1 female) with normal (20/20) or corrected-to-normal vision participated in the present experiment. Participation in this study was wholly voluntary. All methodologies and test procedures, described herein, were approved by the Research Ethics Boards (REBs) of the National Research Council and York University.

\subsection{Apparatus}

A Clinton M20ECD5RE monochrome 19" monitor with short phosphor persistence (p104-100 Hz refresh rate) was used to display experimental stimuli (manufacturer, Clinton Electronics Corporation, IL). A Macintosh G4-dual processor, in conjunction with Matlab 5.2 and Psychophysics Toolbox, was used to control the presentation of the test stimuli and record the observer's responses. Observers were seated at a viewing distance of $1.2 \mathrm{~m}$ with their head stabilized on a chin rest (see Figure 1). The chin rest and chair could be adjusted to accommodate variability in subject size and height.

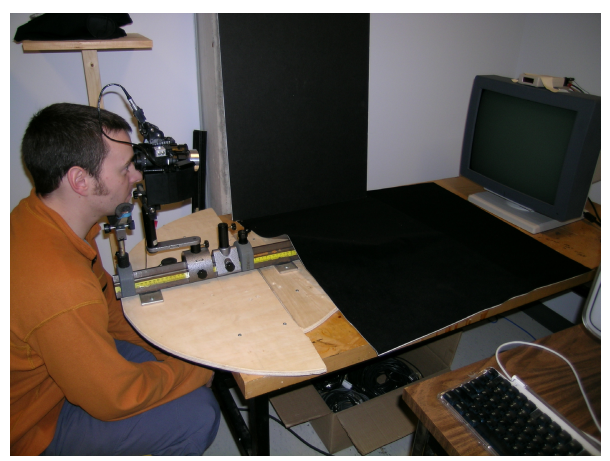

Figure 1. Experimental set-up.

\subsection{Stimuli}

Stimuli consisted of textured squares that were $8.6^{\circ}$ in size. Motion defined targets were created by moving one patch of dots (subtending $4.3^{\circ}$ degrees) in the foreground in a different direction to the dots in the background. The dots were 2 pixels in diameter. If the difference in the speed between the foreground dots and the background dots exceeded a certain threshold, the form of the foreground or background (i.e. in this case a square) was visible. Four different directions of motion-defined square were used, as shown in Figure 2. The side view of these conditions (Figure 2) shows that the perceptual impression is that of a square in the foreground moving in front of the background. The direction of the moving square was randomly selected throughout testing. It is important to note that in all stimulus conditions the stimuli are two-dimensional and do not contain stereoscopic or other cues to depth. These images were rendered in Open GL and displayed at 80\% contrast. Several different dot speeds were used: 20.1, 50.4 100.7, 201.4, 352.45 , and 402.8 arc $\mathrm{min} / \mathrm{second}$. The frame rate was 100 frames per second and stimulus duration was $500 \mathrm{msec}$. For synthetic imagery simulated NVG image noise was added to these motion-defined square stimuli using Thomas et al. (2004) model of a night vision device. ${ }^{9}$ Image noise was added to both the background and foreground of the display. The noise resulted implicitly from the modelled response to a range of input illuminations from full moonlight to cloudy starlight conditions. ${ }^{9}$ Bradley and Kaiser's (1994) radiance values for white paper and green leaves were used to select these input light levels for our high contrast stimuli. ${ }^{12,13}$ The radiance values were used to calculate the 
photons per MCP pore per $10 \mathrm{~ms}$ time frame to be entered into our model and create a realistic range of noise in the image (see Thomas et al., 2004). ${ }^{9}$ Five input light levels were selected as $25,50,100,367$, and 500 photons per pore per frame resulting in five levels of simulated noise. Real imagery was produced by placing broad spectrum Neutral Density (ND) filters in front of the NVG objectives and having subjects view (synthetic) noise free stimulus displays. The ND values were 5.5, 6, and 6.3 producing three levels of input illumination. Input illumination levels in both Real and Synthetic conditions were selected to ensure that a realistic range of values were tested. A final control condition involved having subjects view (synthetic) noise free motion-defined squares that were presented at the same speeds as above. Finally, in both conditions stimuli were viewed monocularly.
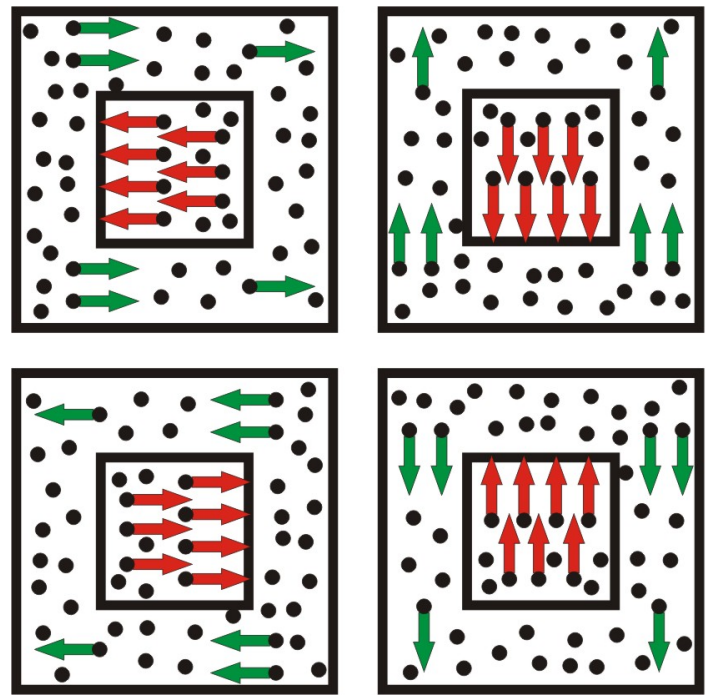

Side View

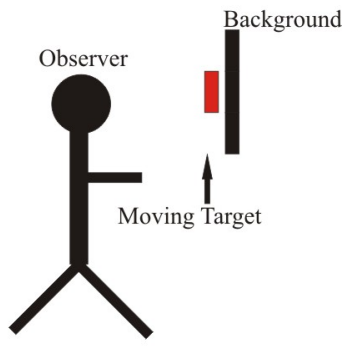

Figure 2. Schematic view of the motion-defined square stimulus configurations used in the present experiment. These views are synthetic imagery, without noise added.

\subsection{Procedure}

On each trial of the Synthetic condition, an observer viewed two consecutive displays interleaved with a brief delay. One presentation contained a motion-defined square with image noise while the other contained image noise and no motion defined square. The order in which squares were presented was randomized across trials. The subject was required to detect whether the stimulus (i.e. the square) was presented in the first or second interval by pushing the keypad number 1 or 2 , respectively. If the observer was uncertain where the square appeared they were instructed to make their best "guess". This psychophysical method of assessing detection performance is known as a Two-Interval Forced Choice Procedure (2-IFC). Since there are two available choices for each test trial, pure guessing resulted in a "chance" level of performance of 50\%. The same procedure was used in the Real imagery condition, with the exception that noise was not explicitly added to the stimuli but was produced by the NVG response to the reduced illumination.

\subsection{Target selection}

Five image noise levels presented at five different image speeds were selected for the Synthetic condition. Similarly, the same five image speeds were use for the Real imagery condition, with three noise levels obtained. These stimuli were then presented in random order according to a method of constants (i.e. with two display presentations on each trial according to the 2-IFC procedure described above). On each trial the observer had to indicate in which interval the stimulus was presented (1st or 2 nd). The total number (or proportion) of correct responses at each stimulus level were 
counted and plotted against the stimulus levels. Testing proceeded until subjects had completed at least 20 trials on each stimulus combination. Performance data were cumulated over sessions. At the end of testing, subjects had accumulated a minimum of 2400 detection trials.

\section{RESULTS AND DISCUSSION}

\subsection{Motion-defined square detection}

For each subject, psychometric functions were obtained by plotting the total percentage of correct choices as a function of image speed for each image noise level. Figure 3A and 4A shows these psychometric functions for the Synthetic condition and Figure 3B and 4B shows performance in the Real condition, for subjects 1 and 2, respectively. Each of the psychometric functions depicted in these figures represents a different Image Noise level. For the Synthetic condition (see Fig 3A, and Fig 4A), the numeric values in the legend represent the modeled input illumination levels (photons per pore per frame). Thus, a smaller value is equivalent to a higher image noise level while a higher value indicates a lower image noise level. In addition, performance for the No noise imagery is plotted in all the graphs (see Fig. 3 and 4). In this case, subjects demonstrate perfect performance across all motion speeds. This demonstrates that the task is trivial when noise is not added to stimuli. It also provides a baseline performance level that can be compared against all conditions in which input illumination is reduced (or effectively noise is added). In keeping with the No Noise condition, uniformly high performance values are noticed at elevated photon input levels in the Synthetic condition (Fig 3A and 4A). Both subjects from both monocular views demonstrate performance decrements across all image speeds when the input light level equals 100 photons per pore per frame or lower. These performance levels are consistently lower than high input illumination levels or when noise is not added to the imagery. Figures $3 \mathrm{~A}$ and $4 \mathrm{~A}$, also show that performance decrements are noticed across all input illumination levels for low speeds. Taken together, these findings are consistent with our previous investigations using synthetic imagery. ${ }^{11}$ Performance for the Real Imagery Condition is plotted in Figure 3B and 4B for subjects 1 and 2, respectively. The numerical values in the legend represent the ND filter values, as the filter density increases the input illumination level decreases (or effectively noise is added). The same general trends as the Synthetic condition are noticed for both subjects across both tubes. As shown in Figures 3B and 4B, a reduction in input illumination (increase in noise) produces a decrease in performance. An overall decrement at both low and high motion speeds is apparent. It is important to note, that there is a similar trend to reduced performance at high motion speeds in the Synthetic condition but it is less pronounced. In summary, these results show that a reduction in input illumination level (i.e. increased noise) for both Synthetic and Real conditions produces decrements in performance. 


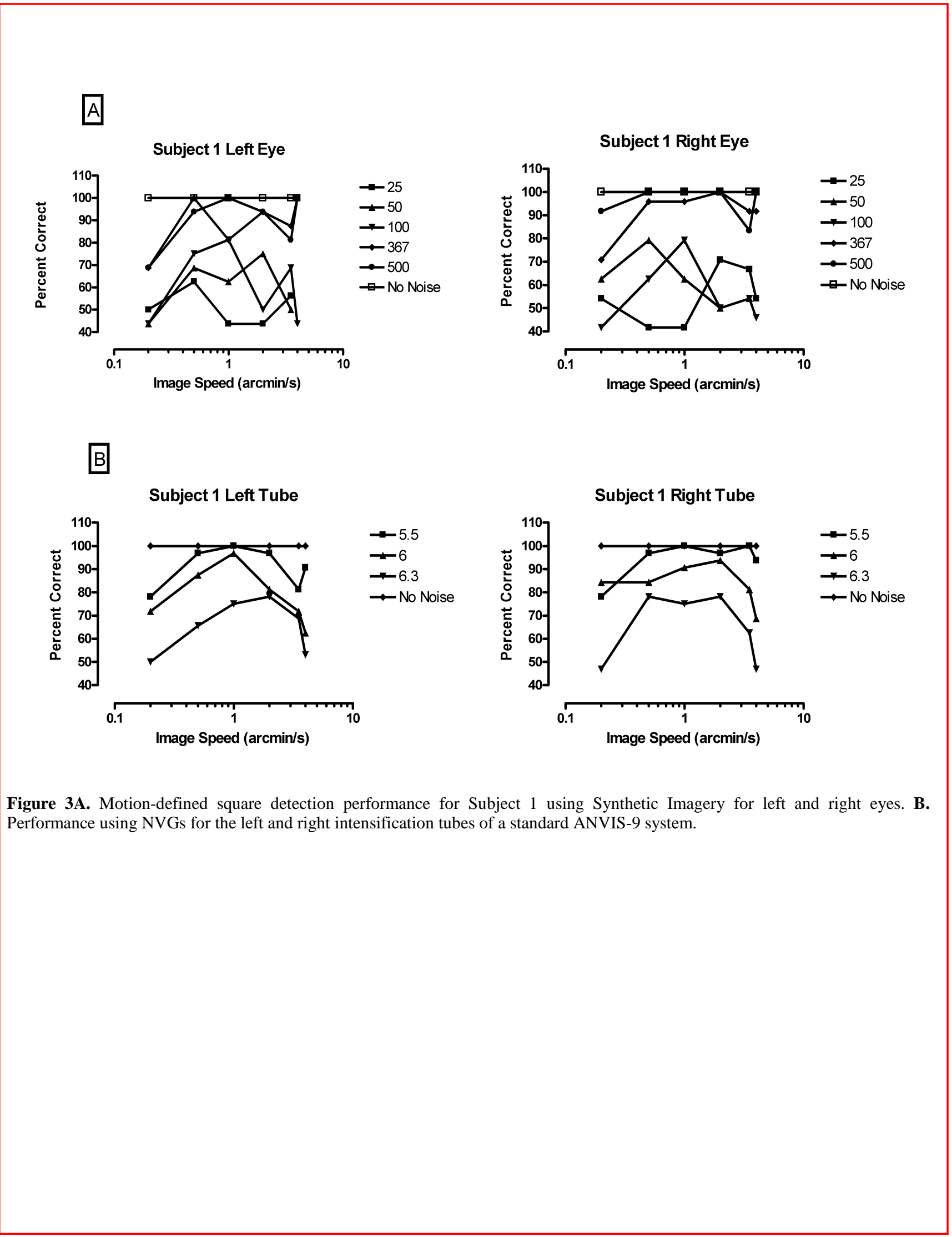



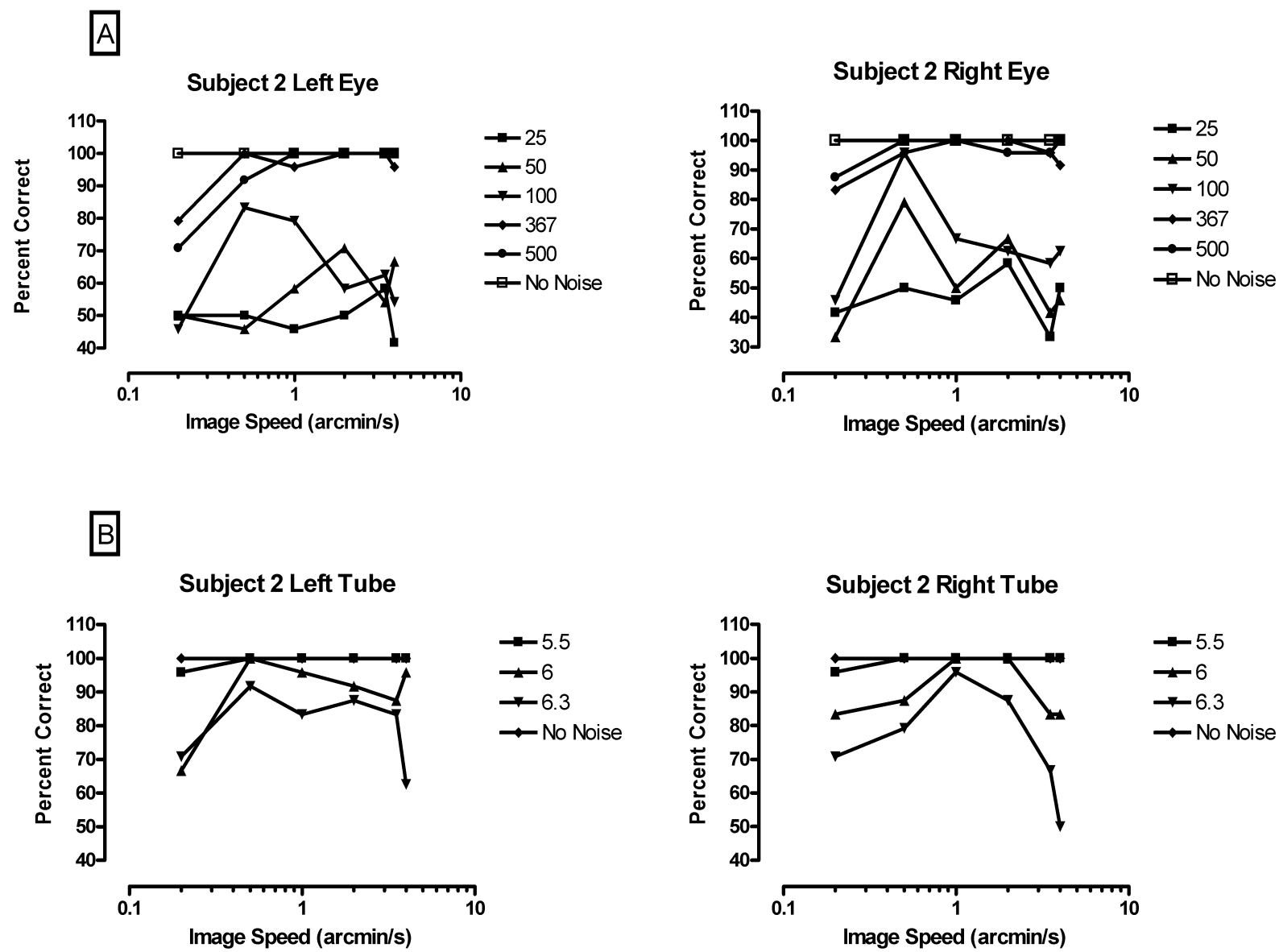

Figure 4A. Motion-defined square detection performance for Subject 2 using Synthetic Imagery for left and right eyes. B. Performance using NVGs for the left and right intensification tubes of a standard ANVIS-9 system.

\subsection{Image noise impairs motion-defined form detection performance}

The detection performance decreased as the image noise was increased (i.e. as input illumination level was reduced) for both Synthetic and Real imagery conditions. Subjects demonstrated a performance decrement for the lowest input light level at all image speeds. These effects were exacerbated at low and high image speeds, particularly in the Real imagery conditions. This is consistent with our previously reported findings using synthetic imagery. The fact that similar results are shown with real NVG image conditions suggests that the synthetic imagery captures relevant features for the task. Thus the results from the Real imagery condition validate and support our model of NVG noise. ${ }^{9}$ 11 


\subsection{Implications for flight: Specifying the influence of NVG image noise on visual perception.}

These results are consistent with anecdotal pilot reports that they have greater difficulty using visual cues under low levels of night time illumination. As we have indicated previously, it is difficult to predict which particular flight tasks might be most affected (e.g. formation flight, cruise at altitude, Nap-of-the-earth (NOE) flight, hover or landing). ${ }^{11}$ In fact, the current results show that image noise reduces the effectiveness of motion as a viable cue. Such findings suggest that other visual cues (e.g. form, luminance, disparity etc) may be impaired at low ambient illumination when goggles have the highest gain settings, consequently producing the highest noise levels. Under these impoverished visual conditions, a broad range of visual mechanisms are likely affected. As a consequence, image noise probably affects flight performance. For example, it may influence the pilot's ability to do collision avoidance and route planning during low-level NOE flight. To address these possibilities it is necessary to examine the impact of image noise on other visual mechanisms. A better understanding of image noise on both visual function and flight performance is required to provide operators with training and other solutions under low ambient illumination levels. By conducting such studies, an empirical database can be established that will allow us to predict the impact of image noise on overall human performance when wearing NVGs.

\section{CONCLUSIONS}

In summary, the capacity to detect motion-defined form decreases as the image noise increases (e.g. due to input light level decreases). These effects are exacerbated at slow and fast image speeds. In other words, as the image speed of a motion-defined form is decreased or increased, an observer requires more input illumination (effectively less image noise) to detect it. These results support pilot reports of difficulty flying under low ambient illumination levels and may be a factor in the increased incidence of helicopter accidents at night. Training protocols based on an understanding of NVG visual performance may reduce the frequency of such accidents. Future investigations will examine the impact of NVG image noise on other visual mechanisms. 


\section{REFERENCES}

1. S.J. Durnford, Crowley, J.S., Rosado, N.R., Harper, J., Deroche, S. "Spatial disorientation: A survey of U.S. Army helicopter accidents 1987- 1992 [Final Report]." Army Aeromedical Research Laboratory Technical Report, Report number AS-A298147, Fort Rucker, AL, 1995.

2. M.G. Braithwaite, Douglass, P.K., Durnford, S.J, \& Lucas, G. (1998). "The hazard of spatial disorientation during helicopter flight using night vision devices. "Aviation, Space and Environmental Medicine, 69, 1038-1044, 1998.

3. S. Jennings, Craig, G. "Theoretical issues relevant to helmet-mounted display attitude symbology." Society of Photo Optical Instrumentation Engineers (SPIE) Proceedings, 4021, 294-301, 2000.

4. Rivamonte, A "Resolution and signal-to-noise measurement U.S. Army night vision goggles", Proceedings of SPIE: Helmet-Mounted Displays II, Lewondowski, R.J. (ed.), Vol. 1290, pp. 206-215, SPIE, Orlando, FL, 1990.

5. R.L.Glasgow, Marasco, P.L., Havig, P.R., Martinsen, G.A., Heft, E.L. "Psychophysical measurements of nightvision goggle noise." Society of Photo-Optical Instrumentation Engineers (SPIE) Proceedings, 5079-18, 2003.

6. F.H. Durgin, Proffitt, D.R., "Perceptual Response to visual noise and display media (Final Report, 1 JUL. 1992-30 JUN. 1993) [Final Report]". Report Number NASA NAG2-814, 1-12.

7. F.H. Durgin, Proffitt, D.R., "Perceptual adaptation in the use of night vision goggles (Final Technical Report, JUN. 1991-MAY 1992) [Final Report]”. Report Number NASA NAG2-721, 1-21

8. Riegler, J.T., Whiteley, J.D., Task, H.L., Schueren, J. "The effect of signal-to-noise ratio on visual acuity through night vision goggles (Interim report, October 1989-September 1990)" NTIS Accession no. AD-A260 579.

9. Thomas, P., Allison, R., Jennings S, Yip, K., Savchenko, E., Tsang, I., Macuda, T., Hornsey, R. "Synthetic Imagery for Night Vision Devices." Society of Photo-Optical Instrumentation Engineers (SPIE) Proceedings, 5442-25, 2004.

10. Reis, G., Marasco, P.L., Havig, P.R. and Heft, E.L. "Psychophysical measurement of night vision goggle noise using a binocular display" Society of Photo-Optical Instrumentation Engineers (SPIE) Proceedings, 5442-13, 2004

11. Macuda, T., Allison, R, Thomas, P., Craig, G. \& Jennings, S. "Detection of motion-defined form under simulated night vision conditions." Society of Photo-Optical Instrumentation Engineers (SPIE) Proceedings, 5442-36, 2004

12. Bradley, A., \& Kaiser, M.K. (1994). "Evaluation of visual acuity with Gen III night vision goggles." Ames Research Center. Moffett Field, CA, NASA report no. N94-23974, 1994.

13. RCA Electro-Optics Handbook (1974). Electro-optics handbook technical series EOH-11. Lancaster: RCA Corp, 1974.

ACKNOWLEDGEMENTS This research was supported by the Canadian Department of National Defence, The Center for Research in Earth and Space Technology (Crestech), Natural Sciences and Engineering Research Council (NSERC), Transport Canada and the Flight Research Laboratory of the National Research Council. We also thank Captain Denis Tang (Canadian Department of National Defence) for useful expert editorial comment and assistance with this project. 
Please verify that (1) all pages are present, (2) all figures are acceptable, (3) all fonts and special characters are correct, and (4) all text and figures fit within the margin lines shown on this review document. Return to your MySPIE ToDo list and approve or disapprove this submission.

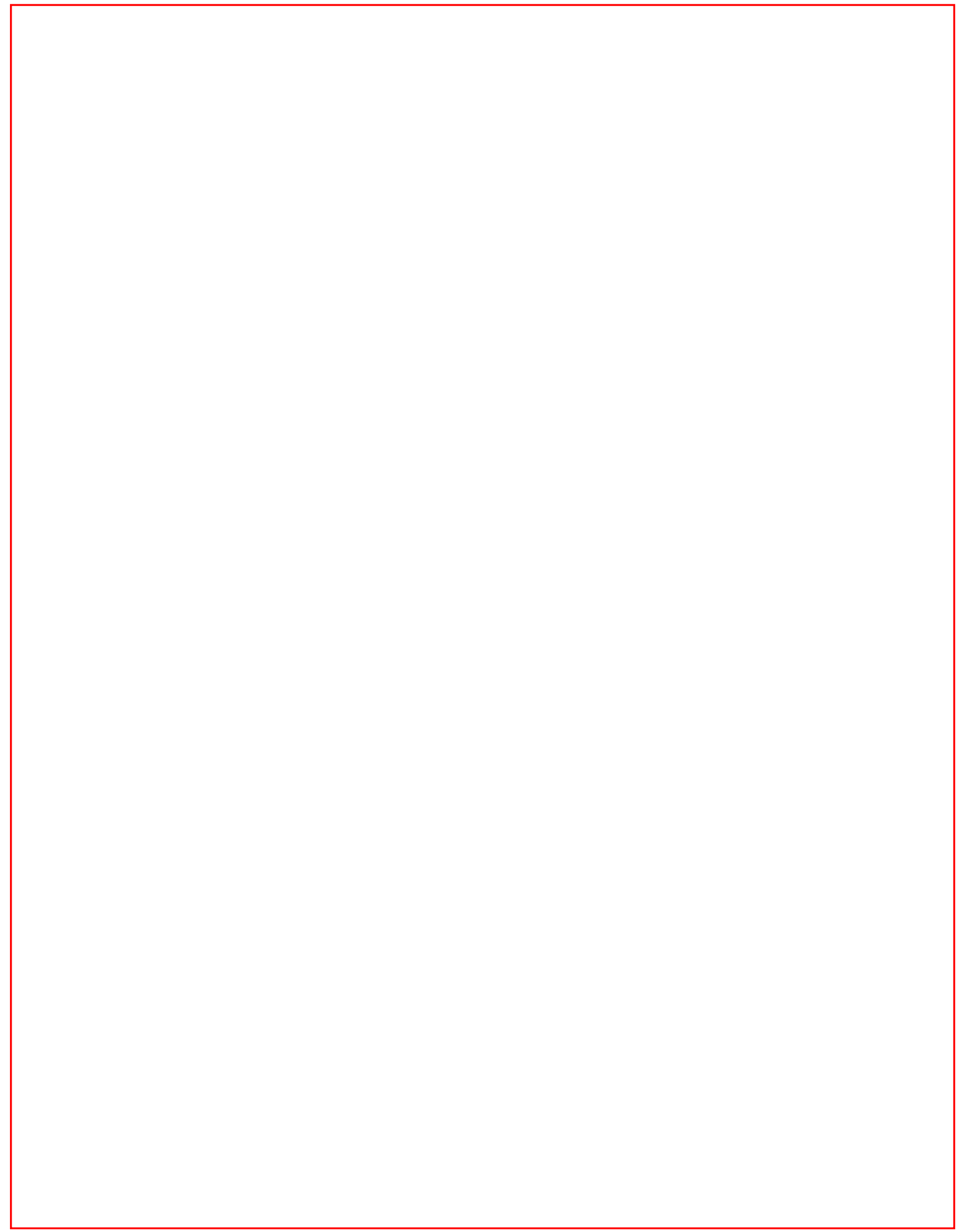

5800-2 V. 1 (p.9 of 9) / Color: No / Format: Letter / Date: 2005-04-11 11:42:56

SPIE USE:___ DB Check, ___ Prod Check, Notes: 